\title{
ASPECTOS CONTABLES Y TRIBUTARIOS DEL DEVENGO BAJO EL ALCANCE DE LA LEGISLACIÓN TRIBUTARIA PERUANA
}

\author{
ACCOUNTING AND TAX ASPECTS OF EARNINGS UNDER THE SCOPE OF THE \\ PERUVIAN TAX LEGISLATION
}

Bill Frank Oscanoa Ponce Ernst \& Young consultores SRL Lima, Perú

ORCID: https://orcid.org/0000-0002-8781-9363 Correo electrónico: bill.oscanoa@pe.ey.com

Ronald Studer Levano Huamaccto Ernst \& Young consultores SRL

Lima, Perú

ORCID: https://orcid.org/0000-0002-5888-8300

Correo electrónico: ronald.levano@pe.ey.com

\section{RESUMEN}

Objetivo: Describir técnicamente los aspectos contables y tributarios del devengo bajo el alcance de la legislación tributaria peruana e identificar una tendencia en la interpretación de esta en el ámbito tributario para su aplicación en la misma. Método: La investigación fue de tipo cuantitativo, con corte transversal, no experimental y de alcance descriptivo. La muestra estuvo conformada por las diversas conclusiones del Tribunal Fiscal por el periodo 1997 al 2017. Resultados: Un 68\% de resoluciones emitidas por el Tribunal Fiscal considera un concepto contable en comparación al $32 \%$ que toma un concepto jurídico para resolver la controversia que enfrenta el devengo. Conclusión: Existe una tendencia significativa en el uso del concepto contable por parte del Tribunal Fiscal entre los años 1997 al 2017 para su aplicación en la Ley sobre el Impuesto a la Renta, dado que siete de cada diez casos fueron resueltos bajo un concepto contable del devengo; por ello, fue necesaria la incorporación de un concepto de devengo en la Ley sobre el Impuesto a la Renta, cuya vigencia es a partir del ejercicio 2019 con la finalidad de mitigar dicha controversia dada en los años anteriores.

Palabras clave: Devengo; Tribunal Fiscal; contribuyentes; Impuesto a la Renta; NIIF 15.
[Recibido: 19/04/2021 Aceptado: 23/08/2021 Publicado: 31/08/2021]

\begin{abstract}
Objective: Describe technically the accounting and tax aspects of the accrual under the scope of the Peruvian tax legislation and identify a trend in the interpretation of the matter in the tax field for its application therein. Method: The research was quantitative, cross-sectional, non-experimental and descriptive in scope. The sample was made up of the various conclusions of the Tax Court from 1997 to 2017 . Results: $68 \%$ of the resolutions issued by the Tax Court consider an accounting concept compared to $32 \%$ that take a legal concept to resolve the controversy facing the accrual. Conclusion: There is a significant trend in the use of the accounting concept by the Tax Court from 1997 to 2017 for its application in the Income Tax Law, given that seven out of ten cases were solved under an accrual accounting concept; because of this, it is necessary to incorporate an accrual concept in the Income Tax Law, whose validity is as of fiscal year 2019 in order to mitigate said controversy that was given in the previous years.
\end{abstract}

Keywords: Accrual; Tax Court; Taxpayers; Income Tax; IFRS 15. 


\section{INTRODUCCIÓN}

El estudio se enfoca en la descripción técnica de los aspectos contables y tributarios del devengo bajo el alcance de la legislación tributaria peruana, con el objetivo de identificar una tendencia en la interpretación de esta en el ámbito jurisprudencial dada por el Tribunal Fiscal durante los años de 1997 a 2017.

En ese sentido, esta investigación busca responder la siguiente pregunta: ¿Existe una tendencia en la interpretación del Tribunal Fiscal (1997-2017) respecto al concepto de devengo?, y bajo ese escenario, la hipótesis planteada es: Existe una tendencia significativa en el uso del concepto contable del devengo. Para tal efecto, se exponen los resultados estadísticos recopilados de los veredictos del Tribunal Fiscal promulgados en los años de 1997 a 2017.

En esa línea, resulta adecuado precisar que la misma Administración Tributaria ha tenido una dualidad de criterios a través de los diversos informes: $\mathrm{N}^{\circ}$ 093-2001, 2652001, 321-2002, 265-2004, 044-2006, 130-2010, 032-2011, 085-2009, 133-2012, 42-2012, 001-2014, 068-2014, 1732016 y 030-2017, 089-2008, 048- 2010, 097-2010.

Asimismo, Durán y Mejía (2017) señalan que el devengo en la legislación tributaria:

Ha desarrollado significativamente los alcances, en torno a un tributo específico (el IR), con un fuerte contenido contable y en el marco de la imputación a un periodo determinado de las rentas y gastos relativos a los contribuyentes domiciliados generadores de rentas empresariales. (p. 2)

Ahora bien, en contra peso, resulta necesario resaltar lo expuesto por Reig (1961), quien afirma que el devengado es un concepto que reúne los siguientes alcances:

(i) Requiere que se hayan dado los hechos principales que genera el derecho u obligación del ingreso o gasto, (ii) Requiere que la acreencia al ingreso no esté supeditado a un requisito suspensivo que deje sin efecto tal derecho y, por último, (iii) No requiere que exista condiciones para el pago, plazo o monto no determinado. (p. 49)

En línea a ello, García Mullin (1978) indicó lo siguiente: "En el devengado solo es relevante una disponibilidad jurídica y no una disponibilidad efectiva del redito" (p. 49). Por lo tanto, y en concordancia con el análisis cualitativo del devengo jurídico de estos autores, resulta importante mostrar que sobre la base del presente estudio cuantitativo soportada en los veredictos del Tribunal Fiscal, se logró divisar una tendencia por el devengo contable.
A tal efecto, la investigación propuesta se ha dividido en cinco partes. La primera establece los aspectos contables del concepto de devengo. En la segunda, los aspectos tributarios del concepto de devengo centrado en la controversia existente. En la tercera, se describe método empleado; la cuarta detalla los resultados. Finalmente, en la quinta se establece la discusión.

Como punto inicial relacionado con los aspectos contables del concepto de devengo, es relevante especificar que el término devengo no se describe directamente en las normas contables sobre los ingresos, sino que se incorpora dentro del Marco conceptual, donde se indica que los estados financieros se basarán en el principio de la acumulación (base de acumulación) reconociendo las transacciones y eventos cuando ocurren.

Por lo tanto, para referir los lineamientos particulares o específicos para considerar devengada una operación en relación con los ingresos ordinarios, se debía recurrir a la Norma Internacional de Contabilidad $\mathrm{N}^{\circ} 11$ (Consejo Normativo de Contabilidad, 2020a) (en adelante NIC 11) y a la Norma Internacional de Contabilidad $\mathrm{N}^{\circ} 18$ (Consejo Normativo de Contabilidad, 2020b) (en adelante NIC 18) como parte del modelo anterior; y ahora, a partir del ejercicio 2019 se deberá considerar a la Norma Internacional de Información Financiera $\mathrm{N}^{\circ} 15$ (Consejo Normativo de Contabilidad, 2020c) (en adelante NIIF 15) como parte del modelo actual.

En ese sentido, la NIIF 15 (Consejo Normativo de Contabilidad, 2020c), como modelo actual, establece una metodología de cinco etapas, las cuales son:

1. Identificar el contrato, 2. Referir a las obligaciones según el contrato, 3. Cuantificar el precio de la transacción según contrato, 4. Distribuir el precio de la transacción entre las obligaciones según el contrato y 5 . Registrar los ingresos cuando se vaya cumpliendo las obligaciones establecidas en el contrato. Siendo ello así , el modelo difere con el modelo anterior, dado que este ultimo se tenía criterios aislados y limitados para el reconocimiento de algunas transacciones que contenían situaciones con múltiples compromisos, lo cual hacían complejo su aplicación; y ahora con el nuevo modelo de ingresos contiene una metodología unificada en una serie de etapas con amplios criterios permite reconocer transacciones muy complejas.

En esa línea, la aplicación del nuevo modelo generará cambios sustanciales en ciertos sectores económicos por la aplicación de nuevos criterios o factores establecidos en 
la etapa 2 de la NIIF 15 (Consejo Normativo de Contabilidad, 2020c).

En ese orden de ideas, los nuevos criterios o factores de apoyo son la integración, dependencia e interrelación descrita en la NIIF 15, las cuales sirven para identificar las obligaciones de desempeño que se encuentran comprometidas con el cliente. Por otro lado, se describe que las obligaciones, según el contrato, pueden referirse de manera directa o indirecta (mencionadas en las políticas, prácticas recurrentes del negocio, entre otros) y que, según señala la norma, deben ser evaluadas al inicio a fin de distinguir si en un contrato se identifica una sola obligación o existen múltiples obligaciones.

Con lo cual, se considera que los bienes o servicios que se brindan serán catalogados como una obligación según el contrato, siempre en cuando se logre coincidir dos criterios NIIF 15 (Consejo Normativo de Contabilidad, 2020c):

1) Los bienes o servicios pueden beneficiarse así mismo o en complemento con otros bienes o servicios disponibles, $\mathrm{y}$

2) La obligación según contrato, que puede ser un bien o servicios, es factible de otrogarlos de forma separada en el contrato; este último criterio es importante, ya que para un bien o servicio sea considerado como una obligación según contrato tiene que ser identificable en el contrato en amplitud; en ese sentido, se identifica la NIIF 15 (International Accounting Standads Board, 2020, párr. 29) considera factores o criterios de apoyo a fin de evaluar el segundo criterio mencionado que en un símil con el modelo anterior de ingresos no los contenía en sus criterios de reconocimiento por tipo de transacción.

Siendo ello así, un punto sustancial del análisis de la NIIF 15 (Consejo Normativo de Contabilidad, 2020c) que genera un cambio de paradigma con el nuevo modelo versus el anterior es descrito a continuación:

Los factores que indican que el compromiso de una entidad de transferir un bien o servicio a un cliente es identificable por separado (...) incluyen, pero no se limitan a los aspectos siguientes: (a) La entidad no proporciona un servicio significativo para la integración del bien o servicio con otros bienes o servicios comprometidos en el contrato dentro de un grupo de bienes o servicios que representan el producto combinado que el cliente ha contratado. (...) (b) El bien o servicio no modifica o ajusta según los requisitos del cliente de forma significativa otro bien o servicio comprometido en el contrato. (c) El bien o servicio no es altamente dependiente o no está fuertemente interrelacionado con otros bienes o servicios comprometidos en el contrato. Por ejemplo, el hecho de que un cliente pudiera decidir no comprar el bien o servicio sin afectar de forma significativa a los otros bienes o servicios comprometidos en el contrato puede indicar que dicho bien o servicio no es altamente dependiente o no está fuertemente interrelacionado con dichos otros bienes o servicios comprometidos.

Conforme a lo anterior, se observa que la incorporación de nuevos factores (el grado de integración, grado dependencia y el grado de interrelación) hacen de la NIIF 15 una norma más integral en comparación con el modelo anterior (NIC 18 y NIC 11). Por lo que se considera que generará situaciones de cambios en el tratamiento contable respecto a determinados sectores, puesto que en la aplicación de los factores que permiten identificar las obligaciones de desempeño, se modificará el tratamiento contable según sea el caso.

De esta forma, se identifican cambios sustanciales en el tratamiento contable en la industria tecnológica y la ingeniería especializada. En ese escenario, los ejemplos de dichos sectores corresponden a productos combinados de bienes y servicios, como son i) el desarrollo de un software con el servicio de instalación incluida, y ii) la venta de bienes especializados con los servicios de instalación a medida.

En lo que respecta a las situaciones de cambio en el tratamiento contable con la NIIF 15, se muestran en la Tabla 1 y 2 los cambios en el tratamiento contable con el modelo actual basado en la identificación de la obligación, desempeño y su momento de reconocimiento de ingresos.

Como se puede apreciar, el tratamiento en el reconocimiento de ingresos es distinto en ambos modelos (ejemplos mostrados en las Tablas 1 y 2), lo que genera un diferimiento de ingresos con el modelo establecido en la NIIF 15 en comparación al modelo anterior de ingresos; debido a que el nuevo enfoque es identificar las obligaciones de desempeño existentes para luego reconocer los ingresos cuando se transfiera el control. Es importante mencionar también que, para los ejemplos mostrados, la entidad crea un activo con un uso alternativo en caso de que el cliente desista de la compra del software.

Sobre lo argumentado, se concluye que la aplicación de la NIIF 15 genera, en ciertos tipos de transacciones, cambios sustanciales de diferimiento en el reconocimiento de 
Tabla 1

Software que incluye un servicio de instalación especializada.

\begin{tabular}{|c|c|c|c|}
\hline \multicolumn{4}{|c|}{ Ingeniería tecnológica: FC110 de la parte C de la NIIF 15} \\
\hline \multicolumn{4}{|c|}{ Aplicación NIC 18} \\
\hline Compromisos & Componentes identificables & 1 & 2 \\
\hline Software & Enajenación de bienes & $\mathrm{x}$ & \\
\hline Instalación & Servicios prestados & & $\mathrm{X}$ \\
\hline \multicolumn{4}{|c|}{ Aplicación NIIF 15 (Factor aplicable integración) } \\
\hline Compromisos & Obligaciones de desempeño & 1 & 2 \\
\hline $\begin{array}{l}\text { Software } \\
\text { Instalación }\end{array}$ & Única & & $\mathrm{X}$ \\
\hline
\end{tabular}

Nota: Se identifica la aplicación del factor integración solo hay una obligación de desempeño (software e instalación) y por tanto en aplicación de la NIIF 15 se contabiliza los ingresos en un momento determinado (año 2)

Fuente: Elaboración propia

Tabla 2

Equipos especializados que incluye el servicio de instalación especializada.

\begin{tabular}{|c|c|c|c|}
\hline \multicolumn{4}{|c|}{ Ingeniería especializada: FC112 de la parte C de la NIIF 15} \\
\hline \multicolumn{4}{|l|}{ Bajo NIC 18} \\
\hline Compromisos & Componentes identificables & 1 & 2 \\
\hline Equipos & Enajenación de bienes & $x$ & \\
\hline Instalación & Servicios prestados & & $x$ \\
\hline \multicolumn{4}{|c|}{ Bajo NIIF 15 (Factor aplicable altamente dependiente) } \\
\hline Compromisos & Obligaciones de desempeño & 1 & 2 \\
\hline $\begin{array}{l}\text { Equipos } \\
\text { Instalación }\end{array}$ & Única & & $x$ \\
\hline
\end{tabular}

Nota: Se identifica la aplicación del factor de dependencia hay una obligación de desempeño (equipo e instalación) y por tanto en uso de la NIIF 15 se contabiliza los ingresos en un momento determinado (año 2)

Fuente: Elaboración propia

ingresos, en comparación con el modelo anterior (NIC 11/NIC 18) que podría acarrear un impacto tributario.

La segunda parte de la investigación aborda los aspectos tributarios del concepto de devengo centrado en la controversia existente, dado que el concepto de devengo bajo el alcance de la legislación tributaria peruana no se encontraba definido en la misma hasta el ejercicio 2018. Por lo que, durante muchos años, se ha convivido con diversas opiniones emitidas por distintos órganos colegiados, tales como son la Superintendencia Nacional de Aduanas y Administración Tributaria (2020), y el Tribunal Fiscal; aplicando en ocasiones un criterio contable, jurídico y mixto (jurídico contable) en relación con el concepto de devengo que recogia la Ley del Impuesto a la Renta. Sin embargo, dicha falta no calificaba como un vacío legal ni el impedimento de la aplicación, ya que por interpretación se daba contenido a dicho concepto.

En la línea de la revisión histórica de los veredictos emitidos por el Tribunal Fiscal, se identifica que no ha existido uniformidad en las opiniones respecto al alcance que se otorga al término devengado.

Por su parte, la Superintendencia Nacional de Aduanas y Administración Tributaria (2020), en el mismo sentido, 
no ha ofrecido un lineamiento uniforme en sus pronunciamientos referidos al concepto de devengo, tal como se muestra a continuación en la Tabla 3:

Tabla 3

Criterio de los Informes de Sunat (2020) y (2021)

\begin{tabular}{ccc}
\hline $\begin{array}{c}\text { Devengo } \\
\text { Jurídico }\end{array}$ & $\begin{array}{c}\text { Devengo } \\
\text { Contable }\end{array}$ & $\begin{array}{c}\text { Devengo } \\
\text { Mixto }\end{array}$ \\
\hline $\mathrm{N}^{\circ} 093-2001$ & $\mathrm{~N}^{\circ} 085-2009$ & $\mathrm{~N}^{\circ} 089-2008$ \\
$\mathrm{~N}^{\circ} 265-2001$ & $\mathrm{~N}^{\circ} 133-2012$ & $\mathrm{~N}^{\circ} 048-2010$ \\
$\mathrm{~N}^{\circ} 321-2002$ & $\mathrm{~N}^{\circ} 142-2012$ & $\mathrm{~N}^{\circ} 097-2010$ \\
\hline
\end{tabular}

Fuente: Elaboración propia con base en la Superintendencia Nacional de Aduanas y Administración Tributaria (2020) y (2021)

En ese sentido, la controversia se hace evidente dada la dualidad de criterios existentes por distintos órganos colegiados tributarios. Por lo cual, para la presente investigación, se han recopilado las resoluciones del Tribunal Fiscal en relación con la discusión del concepto de devengo desde los años 1997 a 2017 para responder la interrogante siguiente: ¿Existe una tendencia en la interpretación del Tribunal Fiscal (1997-2017) respecto al concepto de devengo?

Sin perjucio de lo anterior, es relevante mencionar que la realidad en otros países latinoamericanos, en referencia a la misma problemática en el ámbito tributario, no es distinta. Por ello, a modo de comparación, se describe la situación referida al concepto de devengo en algunos países:

- Argentina: En su Ley de Impuesto a las Ganancias tampoco tiene una definición de devengado y se da la controversia entre considerar "la definición contable" o la definición "agazapada en los textos impositivos".

- Ecuador: Los contribuyentes pagan su impuesto a las ganancias sobre los resultados que arroje su contabilidad, ya que no existe una definición de devengado en sus normas tributarias.

- Colombia: Presenta una referencia expresa a la Contabilidad cuando en su legislación fiscal no tiene una regulación. Sin embargo, cabe precisar que su legislación fiscal tiene importante regulación y definiciones por lo que no llega a la independencia total de la norma contable, tal es el ejemplo, que tiene parámetros para entender el concepto de devengado en su propia legislación.
En dicho escenario de incertidumbre, aunado a la incorporación de la NIIF 15 en el ámbito financiero - que generaba en algunas situaciones un diferimiento de los ingresos financiaramente como se muestra en los ejemplos de la Tabla 1 y 2 -, era necesario que en el Perú se incorporara una propia definición de devengo en la Ley del Impuesto a la Renta.

Siendo ello así, a partir del ejercicio 2019 con la modificación del artículo 57, se incorporó la definición de devengo para fines tributarios con la mención textual mediante el Decreto Legislativo $\mathrm{N}^{\circ} 1425$ que dispone lo siguiente:

Las rentas de la tercera categoría se consideran producidas en el ejercicio comercial en que se devenguen. Para dicho efecto, se entiende que los ingresos se devengan cuando se han producido los hechos sustanciales para su generación, siempre que el derecho a obtenerlos no esté sujeto a una condición suspensiva, independientemente de la oportunidad en que se cobren y aun cuando no se hubieren fijado los términos precisos para su pago. (Decreto Legislativo $\mathrm{N}^{\circ}$ 1425, 2018)

Asimismo, en el mencionado decreto legislativo, se ha incorporado otros supuestos que el legislador ha considerado prudente para diferir ciertos ingresos, siempre y cuando se encuentre en el siguiente supuesto: "No obstante, cuando la contraprestación o parte de esta se fije en función de un hecho o evento que se producirá en el futuro, el ingreso se devenga cuando dicho hecho o evento ocurra" (Decreto Legislativo $\mathrm{N}^{\circ} 1425,2018$ ).

Dicha definición ha generado que el reconocimiento de ingresos para fines tributarios, en ciertos casos, no coincidirá necesariamente con el reconocimiento de ingresos para fines financieros, por ello es importante conocer la diferencia respecto a dicho punto.

Ante ello, se muestra un ejemplo en la Tabla 4 referente a la industria inmobiliaria, en el que una entidad reconoce fiscalmente sus ingresos por el arrendamiento en función a un porcentaje de las ventas de sus arrendatarios de forma bimestral. Bajo este contexto, se observa dos escenarios (i) con las normas tributarias vigentes hasta 2018 y (ii) a partir de 2019, con la nueva definición de devengo incorporado por Decreto Legislativo $\mathrm{N}^{\circ} 1425$.

Como se puede apreciar, el tratamiento tributario de los ingresos es distinto a partir del ejercicio 2019. Esto se debe a que hasta el 2018 el reconocimiento de los ingresos estaba basado en un criterio tributariamente contable; por ende, se devengaba en el mismo momento en el que se registraba contablemente. 
Tabla 4

Ingresos por arrendamiento sujeta a la variable de ventas de forma bimestral del arrendatario

\begin{tabular}{|c|c|c|c|}
\hline \multicolumn{4}{|c|}{ Industria inmobiliaria } \\
\hline \multicolumn{4}{|c|}{ Hasta el ejercicio 2018 (aplicación devengo contable) } \\
\hline Ingresos & Transacciones & & \\
\hline minresos & Iransactiones & 1 & 2 \\
\hline Arrendamiento & Cesión en uso mes 1 & $\mathrm{x}$ & \\
\hline Arrendamiento & Cesión en uso mes 2 & & $\mathrm{x}$ \\
\hline \multicolumn{4}{|c|}{ A partir de 2019 (aplicación devengo tributario) } \\
\hline & & \multicolumn{2}{|c|}{ Año } \\
\hline Compromisos & Obligaciones de desempeño & 1 & 2 \\
\hline Arrendamiento & Cesión en uso mes 1 & & $x$ \\
\hline Arrendamiento & Cesión en uso mes 2 & & $x$ \\
\hline
\end{tabular}

Fuente: Elaboración propia

Sin embargo, a partir del ejercicio 2019 con la nueva definición de devengo, el tratamiento tributario en una situación en donde la contraprestación a recibir se encuentra sujeta a un hecho o evento futuro, como por ejemplo el caso mencionado en la Tabla 4, debe esperarse a que este ocurra para determinar el importe de la contraprestación $y$, en consecuencia, reconocer tributariamente los ingresos. Siendo ello así, se identifica un escenario de diferimiento de ingresos tributariamente con la aplicación de la nueva definición de devengo.

Asimismo, es relevante mencionar que la Superintendencia Nacional de Aduanas y Adminstración Tributaria (2020, 2021) ha emitido un pronunciamiento reconociendo dicha interpretación en el Informe $\mathrm{N}^{\circ}$ 098-2020-SUNAT/7T0000 en el mismo ejemplo mostrado donde ha indicado que:

1. Las ventas netas mensuales del arrendatario constituyen un hecho o evento futuro a que se refiere el tercer párrafo del inciso a) del artículo 57 de la LIR.

2. La contraprestación variable por el arrendamiento determinada en función a las ventas mensuales del arrendatario se considerará devengada en el momento en que se produzcan las indicadas ventas.

3. El devengo del ingreso por las contraprestaciones fijas o variables se efectúa considerando la definición jurídica de dicho concepto prevista en el artículo 57 de la LIR, sin considerar las estimaciones contables del arrendador sobre la posibilidad de no recibir la contraprestación total o parcial.
En ese sentido, es importante tener en cuenta que los parámetros incluidos en la nueva definición de devengo para reconocer tributariamente los ingresos, no necesariamente van a coincidir al momento de reconocer financiaramente los ingresos en aplicación de la NIIF 15.

Finalmente, sobre la base de lo anteriormente mencionado, la incorporación del concepto de devengo en la Ley del Impuesto a la Renta ha establecido una cierta predictibilidad al tratamiento tributario en el Perú a partir del ejercicio 2019 para los ingresos, cerrando la brecha de incertidumbre para las empresas que generan rentas empresariales. Siendo ello así, es importante la participación del Estado en la regulación de las reglas de juego en la sociedad, como North (1993) manifestaba: "Las instituciones reducen la incertidumbre estableciendo una estructura más estable" (p. 16).

Sin embargo, cabe mencionar que aún existen ciertos asuntos que la misma norma no ha aclarado y serán materia de discusión en otras investigaciones.

\section{MATERIAL Y MÉTODOS}

La investigación fue de diseño no experimental puesto que no maniobran las variables de estudio. Asimismo, la investigación fue descriptiva porque tuvo como propósito esbozar la evolución normativa y conceptual de una definición que cambia las pautas en la economía y la tributación, con un enfoque de tipo cuantitativo mediante la evaluación estadística y cuya unidad de análisis fueron las resoluciones emitidas por el Tribunal Fiscal de 1997 a 2017. 
Por lo tanto, a partir de los datos recopilados de la página web del Tribunal Fiscal respecto a los veredictos emitidos de 1997 a 2017 en la interpretación del concepto de devengo, se realizó un análisis descriptivo para identificar la tendencia en la interpretación del concepto de devengo sobre un periodo de tiempo para obtener los resultados y, finalmente, comprobar la hipótesis planteada.

\section{RESULTADOS}

En el Figura 1, se describe la evolución de la interpretación del concepto de devengo en las resoluciones del
Tribunal Fiscal (1997-2017) bajo un criterio contable y jurídico. Es así, como se observa en la Figura 1, que existe una tendencia muy significativa sujeta a la evaluación de la aplicación del concepto contable para la interpretación de esta en el ámbito tributario, siendo una clara tendencia positiva en los últimos años.

Asimismo, como se puede apreciar en la Figura 2, se representa un 68\% de las resoluciones dadas por el Tribunal Fiscal que aplican un concepto contable en comparación al 32\% que considera un concepto jurídico, lo cual contrasta la hipótesis planteada en términos porcentuales.

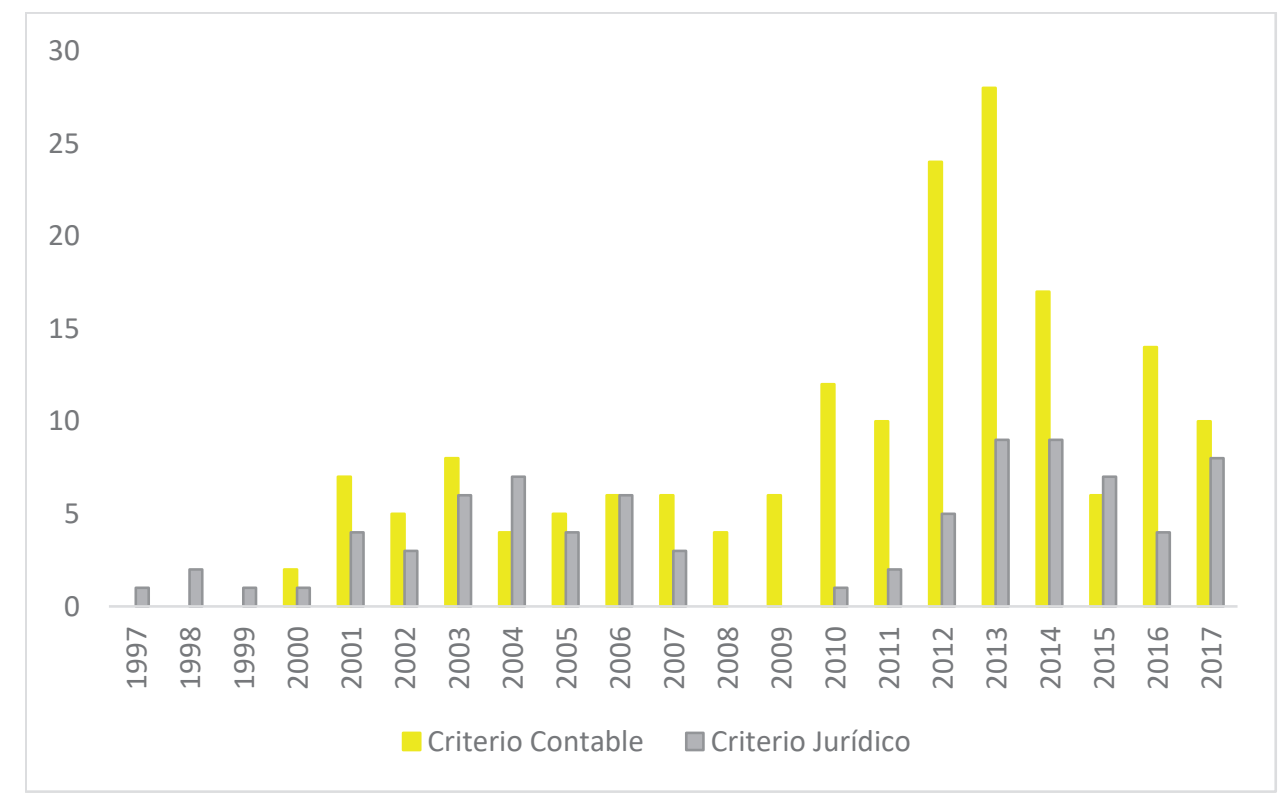

Figura 1. Evolución de los criterios del devengo, periodo 1997-2017

Fuente: Elaboración propia con base en Tribunal Fiscal (2021)

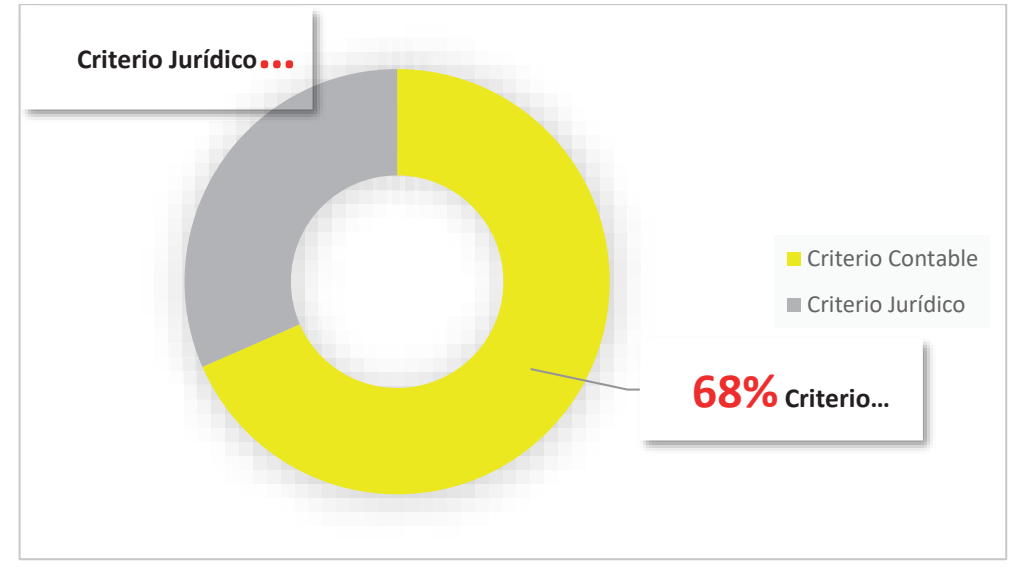

Figura 2. Tendencia del criterio de devengo contable y jurídico

Fuente: Elaboración propia con base en Tribunal Fiscal (2021) 
Por ello, se responde a la interrogante planteada indicando que existe una tendencia significativa en el uso del concepto contable para la interpretación de este en el aspecto tributario hasta el ejercicio 2018, dado que a partir del ejercicio 2019 se aplica la nueva definición de devengo incorporada en la misma legislación tributaria peruana.

\section{DISCUSIÓN}

Del análisis estadístico recopilado (Figuras 1 y 2) de los veredictos del Tribunal Fiscal de 1997 a 2017 referidos al concepto de devengo, se contrasta una tendencia muy significativa en el uso del concepto contable (68\%) en comparación con el concepto jurídico (32\%). Siendo ello así, existía una necesidad en el aspecto tributario de la incorporación de un concepto de devengo en la Ley sobre el Impuesto a la Renta. Por lo que, la emisión del Decreto Legislativo $N^{\circ} 1425$ cumplió dicho fin -aún con ciertas limitaciones-dando así una predictibilidad al tratamiento tributario del reconocimiento de ingresos para los contribuyentes.

Asimismo, es importante notar que el tratamiento contable bajo la NIIF 15, en el cual también ha regido un cambio relevante al momento de reconocer los ingresos mostrados en los ejemplos de la Tabla 1 y 2 , genera un impacto a nivel tributario dado que hasta el ejercicio 2018 existía la tendencia de tomar un criterio contable. No obstante, a partir del ejercicio 2019 con la incorpación del concepto de devengo en la Ley del Impuesto a la Renta, se ha separado el reconocimiento de los ingresos en el ámbito financiero y tributario, tal como se muestra en ejemplo de la Tabla 4, en donde el reconocimiento de ingresos de mandera tributaria puede darse en un momento distinto al reconocimiento de ingresos de forma financiera. Por lo tanto, es importante aplicar los criterios establecidos en la Ley del Impuesto a la Renta respecto al concepto de devengo para reconocer dichos ingresos cuando corresponda bajo la legislación tributaria peruana.

Sin perjuicio de las conclusiones obtenidas, la agenda de investigación para esta temática debería tratar de responder las siguientes inquietudes: ¿Existe una correlación de ingresos y costo bajo los nuevos alcances del devengo incorporado en el Decreto Legislativo $N^{\circ} 1425$ en la prestación de servicios? ¿Genera un impacto la aplicación del Decreto Legislativo $N^{\circ} 1425$ en el Impuesto a la Renta Diferido? ¿El Decreto Legislativo $N^{\circ} 1425$ ha establecido disposiciones transitorias respecto a posiciones tributarias adoptadas donde no existía predictibilidad en el tratamiento tributaria, como por ejemplo ingresos por costos de estructuración?

\section{REFERENCIAS}

Consejo Normativo de Contabilidad. (2020a). Norma Internacional de Contabilidad 11- NIC 11. Recuperado de: https://www.mef.gob.pe/contenidos/conta_publ/ con_nor_co/vigentes/nic/11_NIC.pdf

Consejo Normativo de Contabilidad. (2020b). Norma Internacional de Contabilidad 18- NIC 18. Recuperado de: https://www.mef.gob.pe/contenidos/conta_publ/ con_nor_co/vigentes/nic/18_NIC.pdf

Consejo Normativo de Contabilidad. (2020c). Norma Internacional de Información Financiera 15-NIIF 15. Recuperado de: https://www.mef.gob.pe/contenidos/ conta_publ/con_nor_co/vigentes/niif/NIIF15_2014_ v12112014.pdf

Decreto Legislativo $\mathrm{N}^{\circ} 1425$, Decreto Legislativo que modifica la Ley del Impuesto a la Renta. (2018). Poder Ejecutivo. Diario Oficial El Peruano, 17 de setiembre.

Durán, L., \& Mejía, M. (2017). El concepto de devengado en el Impuesto a la Renta Empresarial peruano. Análisis Tributario, 9, 11-22.

García Mullin, R. (1978). Impuesto sobre la Renta:Teoría y Técnica del Impuesto. Buenos Aires: CIET.

North, D. C. (1993). Instituciones, Cambio institucional y Desempeño Económico. México D.F.: Editorial Fondo de Cultura Económica.

Reig., E. (2010). Impuesto a las ganancias. Estudio teórico práctico de la ley del impuesto a las ganancias a la luz de la teoría general del impuesto a la renta (12a ed.). Buenos Aires: Editorial Errepar.

Superintendencia Nacional de Aduanas y Adminstración Tributaria. (Junio de 2020). Legislación Tributaria. Recuperado de: https://www.sunat.gob.pe/legislacion/ codigo/titulopr.htm

Superintendencia Nacional de Aduanas y Adminstración Tributaria. (Junio de 2021). Legislación Tributaria. Recuperado de: https://www.sunat.gob.pe/legislacion/ oficios/2020/indcor.htm

Tribunal Fiscal. (2021). Búsqueda de precedentes jurisprudenciales por resolución o expediente. Recuperado de: https://www.mef.gob.pe/es/jurisprudencia/busquedas-de-precedentes-jurisprudenciales 